\title{
Performance of the Ultra-High Rate Germanium (UHRGe) System
}

JE Fast, MP Dion, DC Rodriguez, BA VanDevender, LS Wood, ME Wright

December 2013

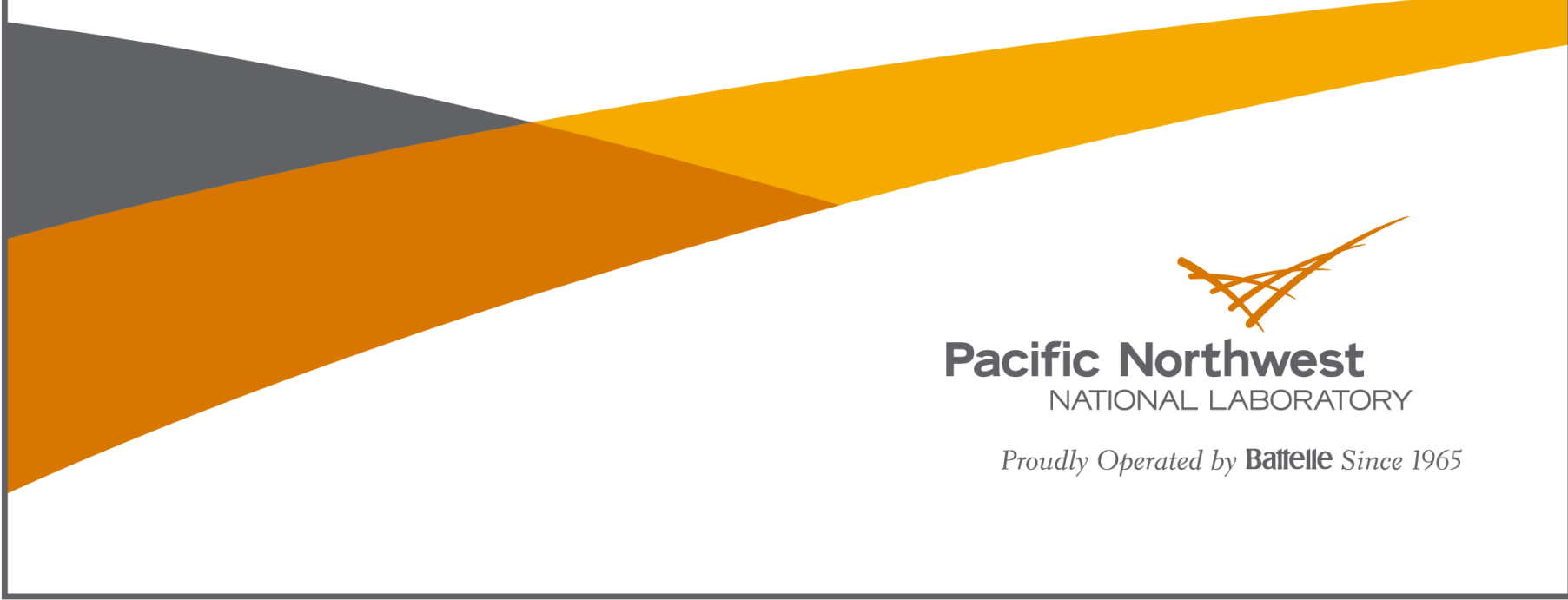




\title{
DISCLAIMER
}

This report was prepared as an account of work sponsored by an agency of the United States Government. Neither the United States Government nor any agency thereof, nor Battelle Memorial Institute, nor any of their employees, makes any warranty, express or implied, or assumes any legal liability or responsibility for the accuracy, completeness, or usefulness of any information, apparatus, product, or process disclosed, or represents that its use would not infringe privately owned rights. Reference herein to any specific commercial product, process, or service by trade name, trademark, manufacturer, or otherwise does not necessarily constitute or imply its endorsement, recommendation, or favoring by the United States Government or any agency thereof, or Battelle Memorial Institute. The views and opinions of authors expressed herein do not necessarily state or reflect those of the United States Government or any agency thereof.

\author{
PACIFIC NORTHWEST NATIONAL LABORATORY \\ operated by \\ BATTELLE \\ for the \\ UNITED STATES DEPARTMENT OF ENERGY \\ under Contract DE-AC05-76RL01830
}

Printed in the United States of America
Available to DOE and DOE contractors from the Office of Scientific and Technical Information,
P.O. Box 62, Oak Ridge, TN 37831-0062;
ph: (865) 576-8401
fax: $(865)$ 576-5728
email: reports@adonis.osti.gov

\begin{abstract}
Available to the public from the National Technical Information Service, U.S. Department of Commerce, 5285 Port Royal Rd., Springfield, VA 22161 ph: (800) 553-6847 fax: $(703) 605-6900$ email: orders@ntis.fedworld.gov online ordering: http://www.ntis.gov/ordering.htm
\end{abstract}

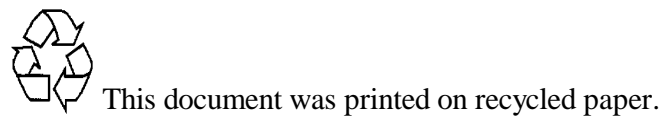




\section{Performance of the Ultra-High Rate Germanium (UHRGe) System}

JE Fast, MP Dion, DC Rodriguez, BA VanDevender, LS Wood, ME Wright

December 2013

Prepared for

the U.S. Department of Energy

under Contract DE-AC05-76RL01830

Pacific Northwest National Laboratory

Richland, Washington 99352 


\section{Executive Summary}

This report describes the final performance achieved with the detector system developed for the Ultra High Rate Germanium (UHRGe) project. The system performance has been evaluated at low, moderate and high rates and includes the performance of real-time analysis algorithms running in the FPGA of the data acquisition system. This performance is compared to that of offline analyses of streaming waveform data collected with the same data acquisition system the performance of a commercial Multi-Channel Analyzer designed for high-resolution spectroscopy applications, the Canberra LYNX.

The UHRGe system was successfully operated at input count rates exceeding $1 \mathrm{Mcps}$ with $40 \%$ live time using a $722 \mu \mathrm{Ci}{ }^{137} \mathrm{Cs}$ source. Energy resolution at high rate was degraded, but remained better than $1.5 \%$ full-width at half maximum (FWHM). This can be compared with the best performance achieved with the fast scintillator $\mathrm{LaBr}$ of $3.6 \% \mathrm{FWHM}$ at $662 \mathrm{keV}$ at $70 \mathrm{kcps}$ with relatively little degradation in resolution at rates up to $2.6 \mathrm{Mcps}$, but with $\sim 2 \%$ gain shifts originating from current flow in the PMTs at high rates [Nocente]. Future system-level improvements, particularly the utilization of a lower capacitance detector electrode configuration, are expected to improve the energy resolution at high rates while faster charge collection times in planar detectors will enable higher live time and throughput. Improvement in throughput is accompanied by only modest compromise in energy resolution.

In addition to system-level investigations of analog and digital signal processing and analysis algorithms performed with a small semi-coaxial detector, the UHRGe project investigated the performance of a novel HPGe pixel detector. This detector is $70 \mathrm{~mm}$ in diameter and $10 \mathrm{~mm}$ thick with 7 electrodes fabricated by $\mathrm{PhDs}$ Co. This was the first detector of this kind ever produced. This detector was able to achieve an energy resolution of $2.87 \mathrm{keV} \mathrm{FWHM}$ at $662 \mathrm{keV}$ for a collimated source over the pixels and $6.75 \mathrm{keV}$ FWHM for uniform illumination of the entire detector surface. The corresponding results for the $59 \mathrm{keV}$ gamma-ray from ${ }^{241} \mathrm{Am}$ are $1.42 \mathrm{keV}$ and $3.13 \mathrm{keV}$, respectively. While we have no direct evidence, we believe that inhomogeneous charge collection (e.g. between low and high field regions), leads to the broadening of the photoelectric peaks for uniform illumination. The electrode configuration was found to be non-ideal for high-rate applications as it generates regions of the detector with long drift times. Thus, while the charge pulses have short durations, as expected from a point contact configuration and desirable for this application, the long drift times make it impossible to time-order energy depositions at high rates making it impossible to build events from Compton scattered events. Alternative electrode configurations have been conceptually developed to eliminate this issue and these will be further studied in the future.

The technology described in this report is being further developed in a new project "PL14NDASpentFuel-PD2Ja_FY14" that will be targeted toward the assay of high burnup fuel and its need for ultra high rate gamma ray spectroscopy to perform isotopic analysis. The Office of Defense Nuclear Nonproliferation Research and Development (NA-22) is also funding the new project. 



\section{Acronyms and Abbreviations}

$\begin{array}{ll}\text { ADC } & \text { Analog-to-digital converter } \\ \text { DAQ } & \text { Data acquisition system } \\ \text { FPGA } & \text { Field-programmable gate array } \\ \text { FWHM } & \text { Full-width at half-maximum } \\ \text { HPGe } & \text { High-purity germanium } \\ \text { PNNL } & \text { Pacific Northwest National Laboratory } \\ \text { UHRGe } & \text { Ultra High Rate Germanium }\end{array}$





\section{Contents}

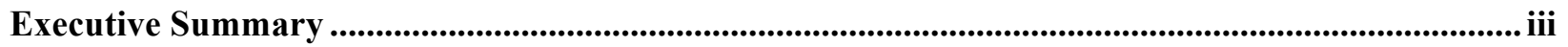

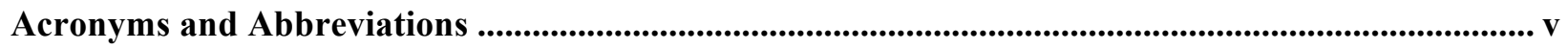

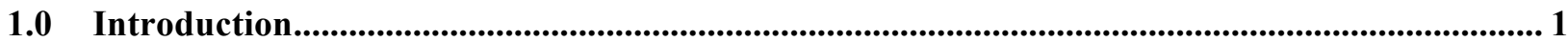

1.1 The Ultra High Rate Germanium Project ….................................................................... 1

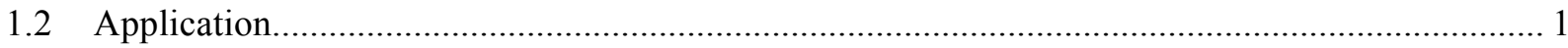

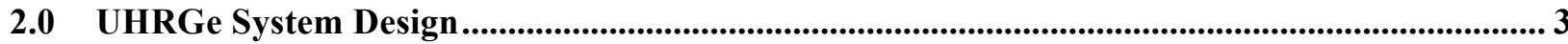

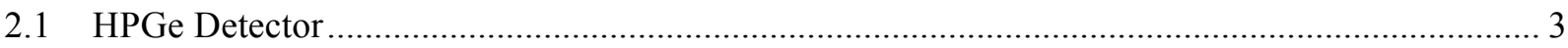

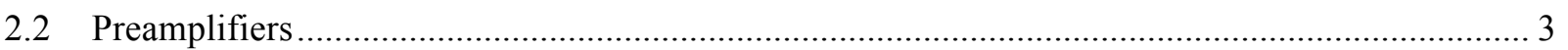

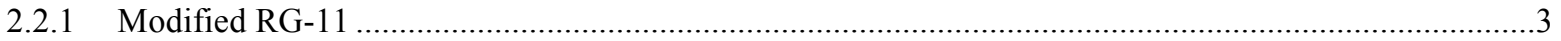

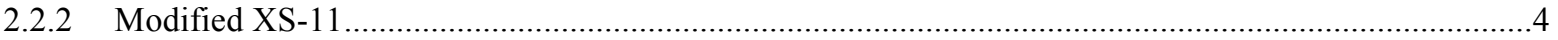

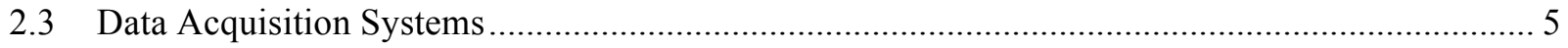

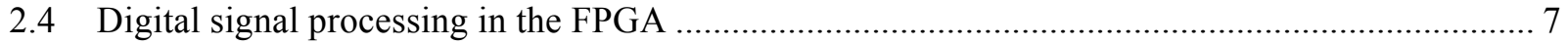

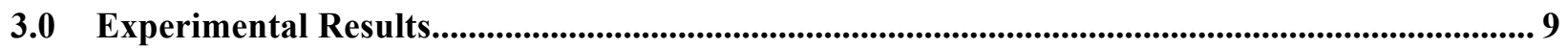

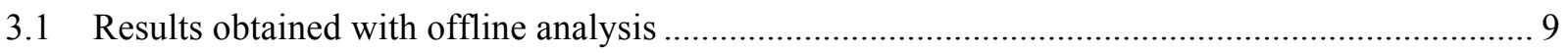

3.2 Results obtained with real time signal processing in the FPGA ............................................... 11

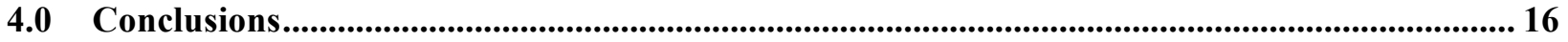

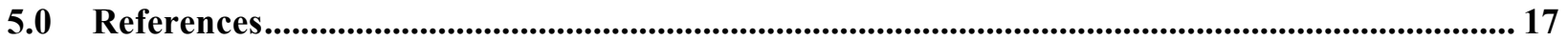




\section{Figures}

Figure 1. Simplified circuit architecture of the XS-11 preamplifier.

4

Figure 2. Measurement demonstrating the high speed and settling precision of the XS-11 - the $55 \mathrm{~ns}$ time constant yields a closed-loop gain of $18 \mathrm{MHz}$ and $180 \mathrm{MHz}$ gain-bandwidth product.

Figure 3. Energy resolution versus shaping time $\mathrm{L}$ of the high purity germanium coaxial detector

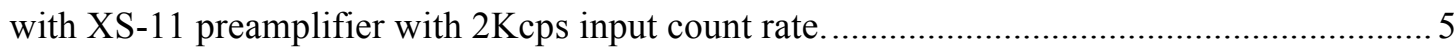

Figure 4. Block diagrams of the DAQ systems investigated....................................................... 6

Figure 5. Simplified block diagram of FPGA variable-length filter implementation....................... 7

Figure 6. Example of multiple energy filters in parallel. Each event is matched with the longest filter that completes before the next event occurs.

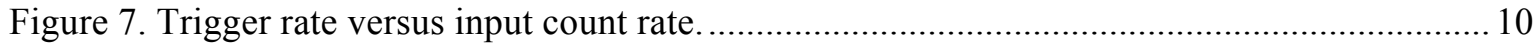

Figure 8. Output rate (to energy histogram) versus input count rate........................................... 10

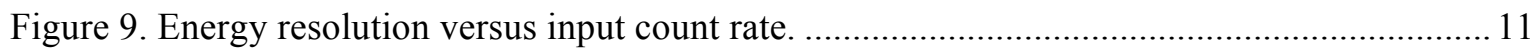

Figure 10. Spectrum at $73.7 \mathrm{kcps}$ input gamma rate, using filter set A...................................... 13

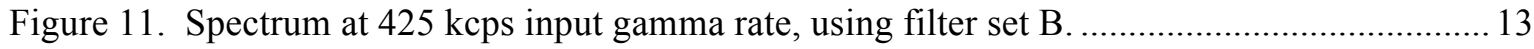

Figure 12. Frequency of use and energy resolution for filter set A at lower rates. The curves represent filters with rise times as shown in the caption with all having the same gap time of $800 \mathrm{~ns}$

Figure 13. Frequency of use and energy resolution for filter set B at higher rates. The curves represent filters with rise times as shown in the caption with all having the same gap time of $800 \mathrm{~ns}$ 


\subsection{Introduction}

\subsection{The Ultra High Rate Germanium Project}

High-resolution high-purity germanium (HPGe) spectrometers are the foundation technology for critical national missions in Emergency Response, Safeguards, and other critical applications. Count-rate limitations of today's HPGe technologies, however, lead to concessions in their use and reduction in their efficacy. Large-volume, very high-rate HPGe spectrometers are needed to enable a new generation of nondestructive assay systems with the target application being the gamma-ray analysis of spent nuclear fuel for both this project and its successor.

The Ultra High Rate Germanium (UHRGe) project goal was to develop technology to operate high purity germanium (HPGe) detectors with high throughput (>85\%) in environments with incoming gamma/x-ray event rates $\mathrm{r} \sim 10^{6} \mathrm{~s}^{-1}$ in a single detector with minimal loss of energy resolution relative to low-rate operation at $\mathrm{r} \sim 10^{3} \mathrm{~s}^{-1}$. The design of such a detector system demands attention to details of the entire signal chain from the detector geometry through the analog and digital electronics and ultimately to the algorithms used to extract the pulse height information and construct energy spectra.

The UHRGe project has developed the analog readout, digital signal processing, and data reconstruction software necessary to operate HPGe at unprecedented rates $(\sim 1 \mathrm{MHz})$. Looking forward from the gains achieved in this work, further improvement would be expected from coupling this high rate capability demonstrated on a monolithic semi-coax HPGe detector to a pixellated (few $\mathrm{mm}$ to $\mathrm{cm}$-scale pads or strips/striplets) HPGe detector. This would eliminate the need for extreme collimation in the measurement system to reduce the count rate, increasing the throughput capability by an additional order of magnitude and opening the possibility to perform imaging that may be of extremely high value. Such a solution would leverage the electronics and signal processing developed in this work to fully utilize the unique characteristics (charge collections time, capacitance) of a pixellated HPGe detector.

\subsection{Application}

There are several safeguards applications that require very high-rate gamma spectrometry with germanium spectral resolution, with prime examples being the assay of spent fuel pins and assemblies to verify burn-up in the presence of an intense background from Cs-137 and other fission products, verification of $\mathrm{U}-235$ mass in $\mathrm{UF}_{6}$ cylinders, and non-destructive assay for quantification of $\mathrm{U}$ and $\mathrm{Pu}$ isotopes in samples of various types. We will discuss the spent fuel assay and non-destructive assay applications here.

One of the challenges in spent fuel assay is to determine Pu isotopic concentrations. This is often done using high-resolution spectroscopy coupled to burn-up calculations. In this application large germanium detectors are used to provide a good peak-to-Compton ratio for the more-penetrating, higher-energy gamma rays, thus minimizing the Compton continuum in the lower energy region of interest and allowing greater depth-of-interrogation in the fuel pin or assembly. In order to operate large HPGe detectors in these intense radiation fields, they are highly collimated, with typical orifice sizes of only a few millimeters. This leads to further degradation of the spectrum from forward scattering in the collimator and importantly, a limited field of view that necessitates mechanical scanning, even for measurements on individual pins $(\sim 1 \mathrm{~cm}$ in diameter). Very high-rate, high-resolution spectrometers would allow a larger field of view and translate to more clearly distinguished peak regions (reduced scatter in collimator). 
These improvements would in turn produce improved accuracy and precision for key isotopic ratios used to verify burn-up calculations, or to directly measure $\mathrm{Pu}$ and $\mathrm{U} \mathrm{x}$-rays.

The second application is in non-destructive assay for Safeguards and other applications. The return signal from active interrogation scenarios for nondestructive assay is often a very brief pulse of high-rate gammas. Because the duty cycle is low, detecting as many return gammas as possible is important. This requirement is in tension with large HPGe systems having poor high-rate capabilities. The proposed solution would provide both the efficiency and high-rate capability to allow HPGe to function in active interrogation scenarios with pulsed interrogation sources. The excellent spectroscopic resolution of HPGe will provide the material and isotopic sensitivity desired in these applications.

In addition to the Safeguards applications of this technology, other communities are interested in highrate, high-resolution gamma-ray spectroscopy. The Consequence Management mission within DOE has an expressed need (NA-42 FY10 call for proposals item CM.6.1) for high-rate germanium capability. In addition to the CM shortcoming, other missions of NA-42 require high rate, high efficiency HPGe detectors for time critical gamma ray analysis of particular scenarios. This mission has consistently pushed the envelope for the largest, fastest, and highest resolution HPGe detectors available. 


\subsection{UHRGe System Design}

The UHRGe detector system consists of a HPGe detector, preamplifier circuit, data acquisition system with on-board digital signal processing, and a backend software suite for further data analysis and user interface.

\subsection{HPGe Detector}

The HPGe detector used in the UHRGe project test bed is a $\sim 40 \%$ relative efficiency p-type semi-coax detector manufactured by Princeton Gamma-Tech (PGT). It was a standard detector and was not itself modified for enhanced high rate performance. The detector element is $62 \mathrm{~mm}$ diameter by $45 \mathrm{~mm}$ long and is housed in a standard vertical dipstick.

The feedback resistor and front-end FET are inside the cryostat and operated cold, typically around 120$150 \mathrm{~K}$. In order to extend the rate capability of the UHRGe system one of the two cryostats was modified by adding a second $2 \mathrm{GOhm}$ feedback resistor connected to another feedthrough pin on the cryostat, allowing the detector to be operated either with the standard 2 GOhm feedback or with 1 GOhm by using the two resistors in parallel via connection external to the cryostat.

\subsection{Preamplifiers}

Preamplifier development was a significant effort for the UHRGe project. The preamplifier development was previously reported in PNNL-22164. The final UHRGe test bed operates two detectors, one instrumented with a modified PGT RG-11 that can operate with feedback voltage swing up to -60V and the other with a custom PNNL high-swing preamp dubbed the XS-11 which can operate with feedback voltages as high as $-100 \mathrm{~V}$.

\subsubsection{Modified RG-11}

The modification to the RG-11 is rather simple using the proprietary schematics available from the manufacturer ${ }^{1}$. The supply rail for the feedback node in the standard circuit is $-24 \mathrm{~V}$ entering through a current regulating diode (CR2). The supply side of this element was lifted from the $-24 \mathrm{~V}$ rail and connected to an external voltage source operating at up to $-60 \mathrm{~V}$. The connection to R34 was cut to prevent this high voltage from propagating to the current sense pin on the DB-9 (preamp power) connector. The voltage that can be applied is limited by the ratings of the current regulating diode, an IN5312, and the transistor Q5, a 2N5087 that are rated at 50V.

\footnotetext{
${ }^{1}$ PGT was purchased by Thermo-Fisher and products are now available through Berkeley Nucleonics at http://www.berkeleynucleonics.com
} 
PNNL-23084

\subsubsection{Modified XS-11}

The PNNL-designed preamplifier developed expressly for UHRGe, the XS-11, demonstrated an energy resolution as low as $2 \mathrm{keV}$ at $662 \mathrm{keV}$ (comparable to performance with the stock RG-11 which achieves $1.8 \mathrm{keV}), \mathrm{a}-100 \mathrm{~V}$ output swing to support rates to 1 million and above, and a 100ns step response - a faster response than the charge collection time of the semi-coax high purity germanium detector in the test bed. This XS-11 rev. 0 was found to have defects. (1) When tuned for high-speed operation, critically damped, there was severe noise peaking at about $5 \mathrm{MHz}$ in certain operating configurations. (2) Due to thermal limitations, the low noise $-16 \mathrm{~V}$ regulator could not sustain much DC offset. (3) The closed-loop charge sensitive amplifier is complicated, being made up of four interacting servo loops, the main loop, the high voltage swing output stage broad-banding loop in cascade with the first amplifier stage and main loop, the remote cold JFET drain capacitance nulling loop nested within the first amplifier stage main loop, and the amplified cascode loop nested within the first amplifier stage and main loop. The latter nested loop oscillated at $85 \mathrm{MHz}$, resulting in a few $\mathrm{mV} 85 \mathrm{MHz}$ sine wave at the amplifier output.

Rev. 1 includes fixes for the above defects, re-designs the output stage for significantly lower noise, and includes a novel ultra-linear ultra-low noise JFET source follower shaper "pick-off" amplifier (similar to an oscilloscope input amplifier but far more linear). A totally new printed circuit board layout was required, which was carefully optimized for the required ultra-high signal integrity and precision high speed settling time, while retaining the $125 \mathrm{~V}$ charge-sensitive amplifier output swing. The charge sensitive amplifier is an entirely discrete transistor design with no integrated circuits as no $>70 \mathrm{~V}$ swing IC amplifiers commercially available are anywhere near as fast as the XS-11.

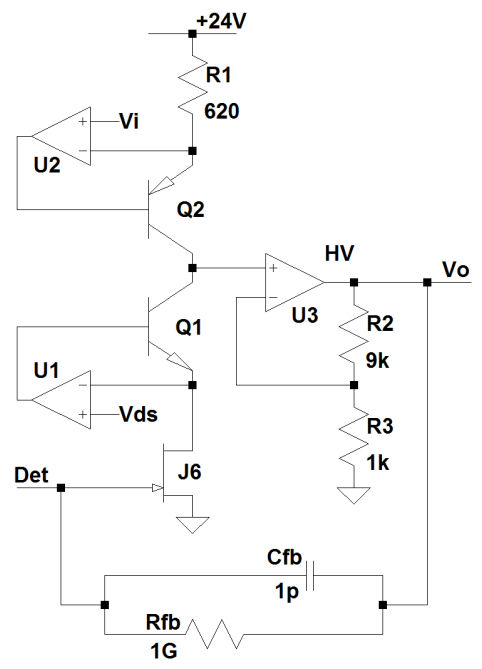

Figure 1. Simplified circuit architecture of the XS-11 preamplifier. 


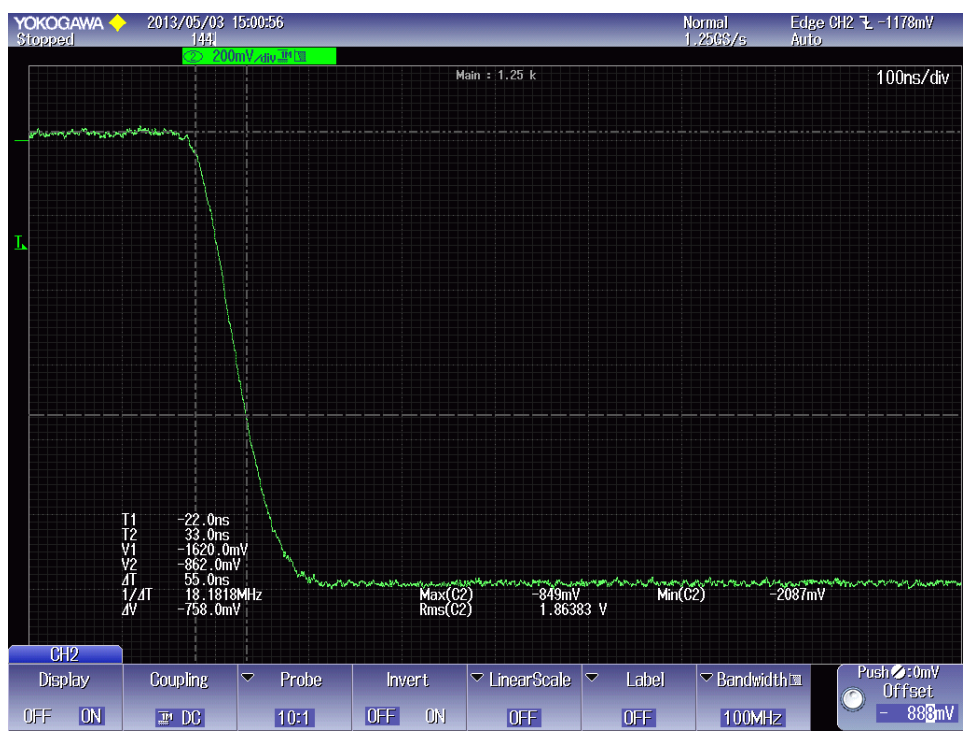

Figure 2. Measurement demonstrating the high speed and settling precision of the XS-11 - the 55 ns time constant yields a closed-loop gain of $18 \mathrm{MHz}$ and $180 \mathrm{MHz}$ gain-bandwidth product.

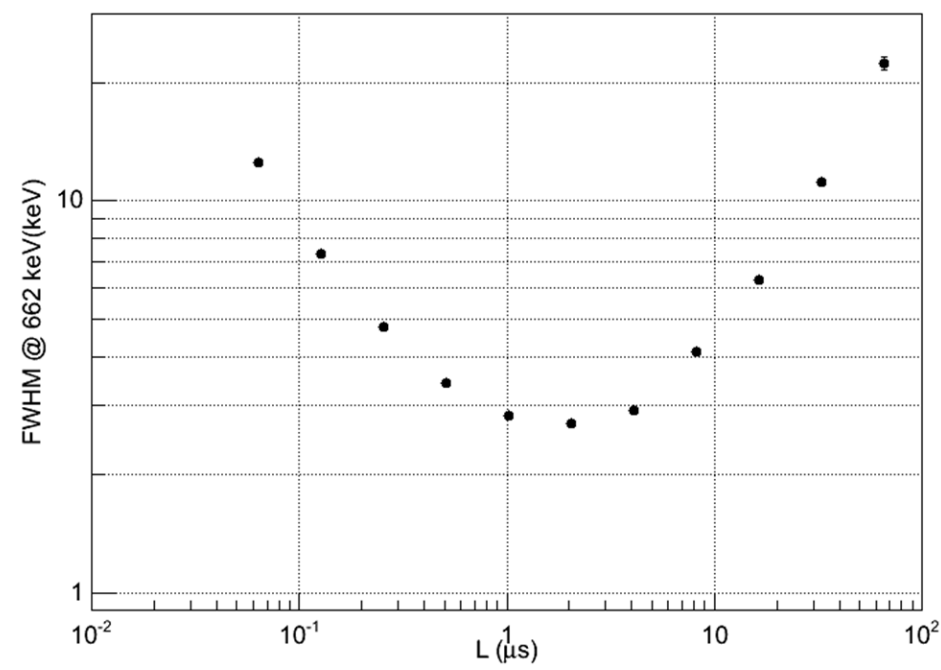

Figure 3. Energy resolution versus shaping time $\mathrm{L}$ of the high purity germanium coaxial detector with XS-11 preamplifier with $2 \mathrm{kcps}$ input count rate.

\subsection{Data Acquisition Systems}

The UHRGe project explored multiple paths for the digitization and digital signal processing hardware. As a reference system for evaluating low-rate performance of analog electronics (preamplifier) designs, a Canberra LYNX multi-channel analyzer was used. 
For high-rate data taking four different digitizers have been investigated, two on the project and two via other work at PNNL that afforded an opportunity to test digitizer performance with the UHRGe detector and preamp system at high rates.

The first system is the Signatec PX14400D PCI Express card that provides continuous $400 \mathrm{MHz}$ sampling of a 14-bit ADC (analog-to-digital converter). This system was found to have numerous serious flaws including injection of a $350 \mathrm{kHz}$ sine wave on the signals with amplitude proportional to DC level setting, a low-resolution DAC controlling the DC level offset resulting in large steps in the output level as the DAC undergoes bit-flips, and missing code errors in the ADC.

The second system is the AlazarTech ATS9626 16-bit, $250 \mathrm{MHz}$ PCI Express waveform digitizer (see Figure 4). While not ideal, this system significantly outperforms the Signatec system and is the main data acquisition platform of the UHRGe test bed. User data filtering is accomplished with a dedicated Altera Stratix III EP3SE260 FPGA. Data is transferred at $1.7 \mathrm{MB} / \mathrm{s}$ to the host computer.

An XIA Pixie-500 Express was tested at rates up to $300 \mathrm{kcps}$ input. At this rate we were able to achieve $30 \%$ live time and energy resolution similar to what we have measured on the Canberra LYNX system at this rate. This system is the foundation that XIA is building on to develop a digitizer for this application under a DNN R\&D sponsored SBIR project.

Finally, we were able to evaluate a 4DSP VP680 VPX card with an FMC108 digitizer module (16-bit, $250 \mathrm{MHz}$ ) system that was obtained as part of a PNNL internally funded Laboratory Directed Research and Development project (see Figure 4). This system performed comparably to the AlazarTech system.
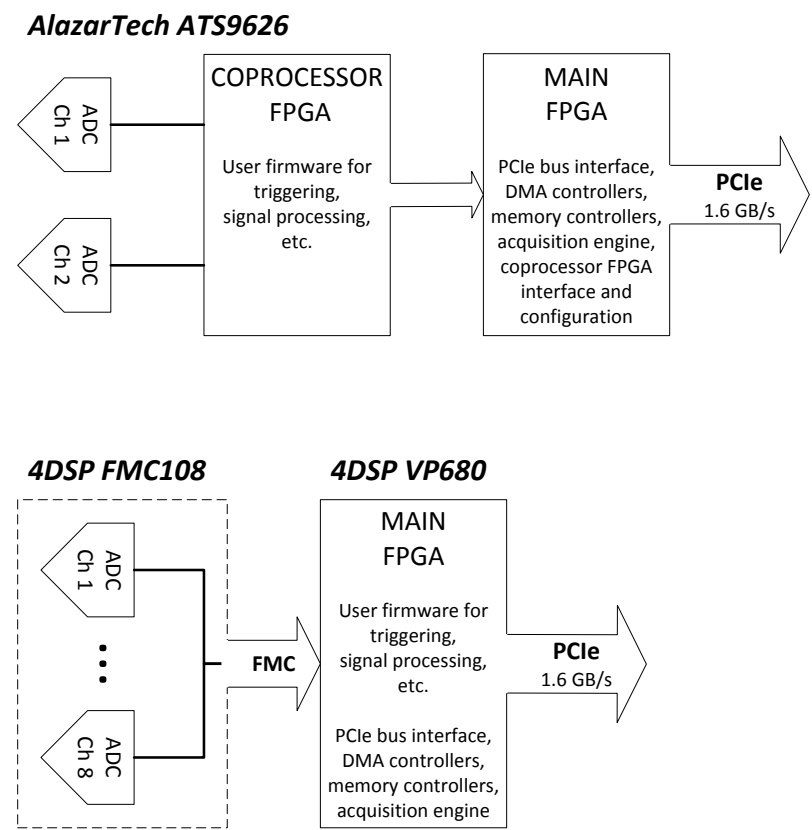

Figure 4. Block diagrams of the DAQ systems investigated. 


\subsection{Digital Signal Processing in the FPGA}

A major emphasis in the signal processing algorithms that were developed with offline analysis through the project lifecycle has been portability to real-time processing in the FPGA that resides on the digitizer board. The analysis techniques, previously reported in PNNL-21895, rely on running a number of trapezoidal filters and then using the outputs to determine various parameters of interest, ultimately the time and energy related to each pulse in the waveform (see Figure 6). The trapezoidal filtering algorithms are standard methods developed by Jordanov and Knoll [Jordanov]; a more complete description of the FPGA implementation is being submitted for publication in IEEE Transactions on Nuclear Science [Wood].

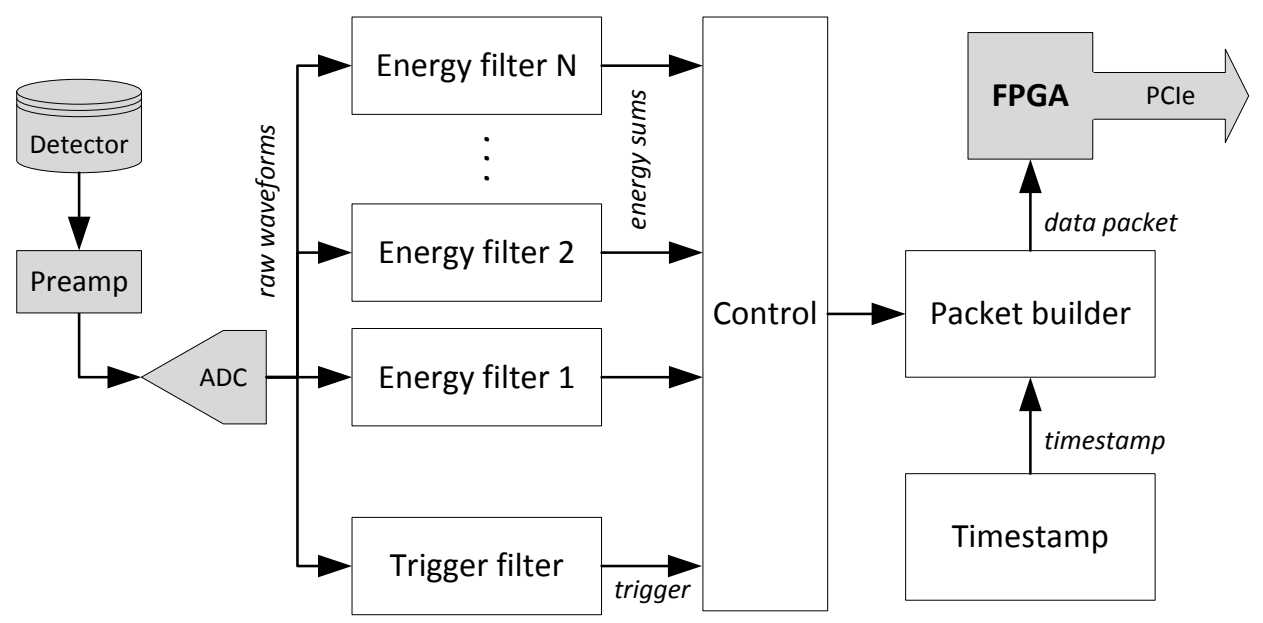

Figure 5. Simplified block diagram of FPGA variable-length filter implementation.

A fast "trigger" filter is run to identify pulses in the waveform. This filter is a simple triangular filter (trapezoid with rise time $=50-100 \mathrm{~ns}$ and gap time $=0$; see [Jordonov] for definitions). The output of this filter is also used to determine the pulse time by measuring the time the waveform passes two different low level discriminators and back projecting using the slope between these points to extract $\mathrm{t}=0$. A series of multiple energy filters with minimal gap time for the charge collection time of the HPGe detector (800 ns for the semi-coax used in these studies) and varying rise times are run in parallel on the incoming data stream from the ADC. The energy filters are run with rise times from as short as $16 \mathrm{~ns}$ up to 16.384 us. The filter with the longest rise time that can be fit between the preceding pulse and the subsequent pulse is used to determine the energy for the pulse being evaluated (see Figure 6). The longest filter's rise time is set by the optimum rise time determined by analysis of each individual filter. A typical optimum length for low-rate operation is between 4 and 16 microseconds, depending on the low-frequency noise environment the detector is operating in. The optimum length is reduced to as little as $512 \mathrm{~ns}$ at very high rates, for reasons not yet understood.

The FPGA then outputs time-stamped list-mode data containing the time and energy determined as described above. In addition, the data stream may contain the current determination of the DC level for monitoring purposes, each of the energy filter values, trigger filter time over threshold which is indicative of pulse rise time and can be used to look for "trigger pileup" events, and other pulse shape data of interest. 
PNNL-23084

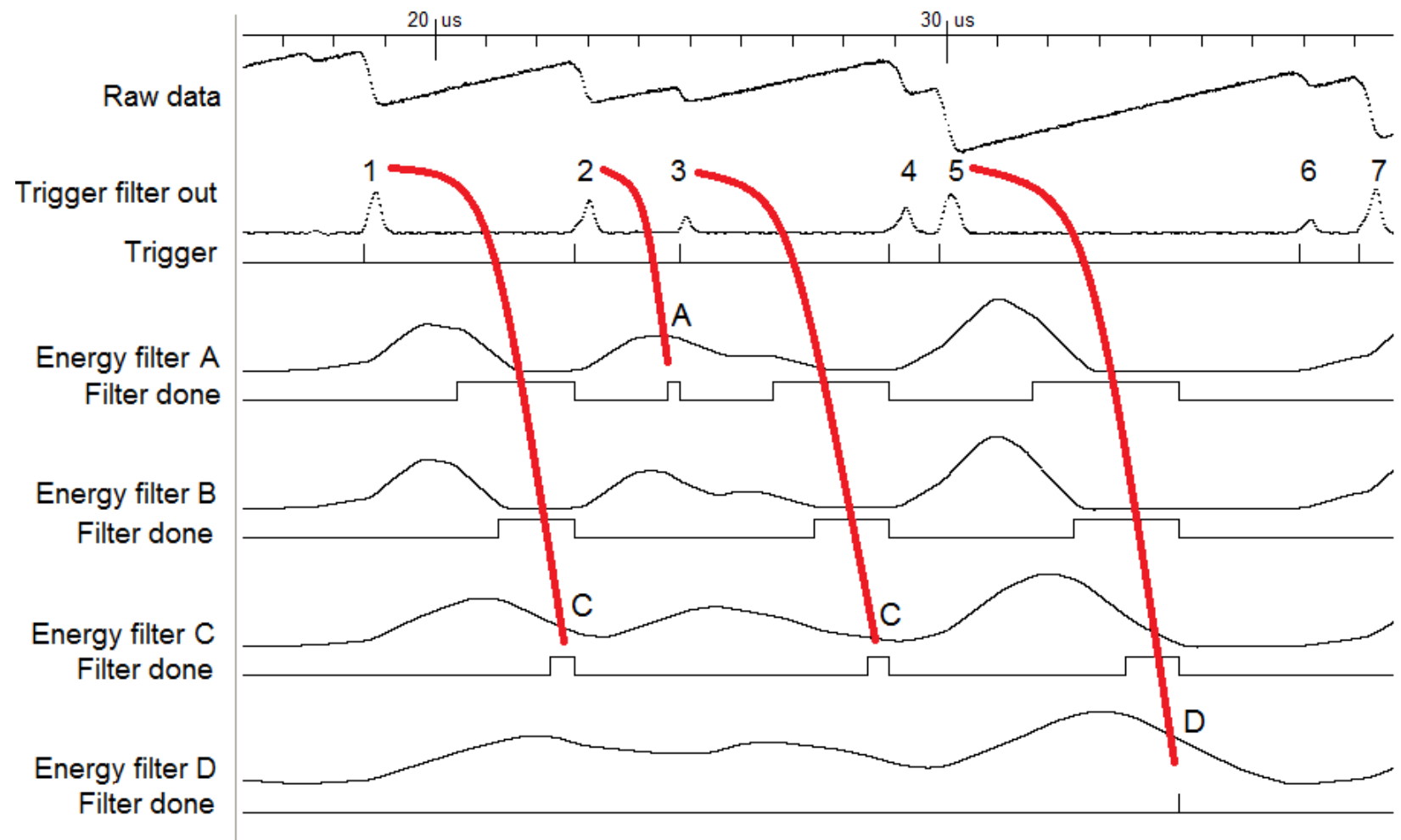

Figure 6. Example of multiple energy filters in parallel. Each event is matched with the longest filter that completes before the next event occurs. 


\subsection{Experimental Results}

\subsection{Results obtained with offline analysis}

The algorithm described above was implemented in offline software to allow performance evaluation to proceed in parallel with hardware implementation. Preamplifier voltage output waveforms were digitized by the AlazarTech unit in long $(1 \mathrm{~ms})$ continuous segments and recorded to disk. The analysis software is realized in $\mathrm{C}++$ and built upon libraries that are standards in the analysis of nuclear physics data. At the lowest rates tested here ( $\sim 100 \mathrm{kcps})$ there are on average 100 events in each $1 \mathrm{~ms}$ segment. Differences in results due to the non-continuous nature of the data are expected to be on order of $1 \%$ or less with respect to expected hardware implementation results, caused by the loss of the first and last event in each segment. These two events cannot be analyzed in the software analysis because the algorithm requires for every pulse, the previous and next pulses, one of which does not exist in the data for the first and last pulses in the segment.

Data were collected by placing a $722 \mathrm{uCi}{ }^{137} \mathrm{Cs}$ source at varying distances from the HPGe detector. Distances were approximately $1-20 \mathrm{~cm}$ from the top of the cryostat. The nominal true event rate for each data set is taken to be the value of $r$ that maximizes the probability of the fit of a function $r e^{-r \Delta t}$ to a histogram of the data binned by $\Delta t$, where $\Delta t$ is the time between consecutive triggers. The given functional form is the expected distribution for the random decays of a radioactive source. It is independent of the pileup rejection criterion to good approximation at lower rates. The approximation begins to break down at our highest rates due to the increased likelihood of multiple event pileups. The independent variable "Input Rate" in all of the plots below is the value of $r$ determined in this way.

The three plots below are results for operation at incoming event rates in the range of $0.2-1.1 \mathrm{Mcps}$. Data are analyzed for four different values of fast trigger rise times $l$ as denoted in the legends. Figure 7 shows the rate of fast trigger occurrences. It rises linearly with the input rate and is improved as the trigger rise time is decreased. The linear rise would be expected to continue up to almost $5 \mathrm{Mcps}$ input rates for our paralyzable pile-up rejection scheme. The improvement with decreasing trigger rise time is attributed to the shorter pile-up resolution window of the shorter trigger pulse. The improvement is most pronounced at the highest rates where pileup during this short window is most probable. Figure 8 shows the rate at which pulses are successfully analyzed and booked into a spectrum of pulse-heights. It rises linearly for low rates and approaches a plateau for our highest rates. The plateau is caused by the increased probability that even the shortest energy filter in our algorithm does not complete. The length of our shortest filter is dominated by the need to accommodate slow rising pulses in our large HPGe detector. A faster detector would plateau with a higher spectral rate at a higher input rate, as discussed above. The ratio of Spectral Rate to Input Rate is what we usually refer to as "throughput". Figure 9 shows the energy resolution versus rate. Energy resolution is measured as the full-width at halfmaximum (FWHM) of the ${ }^{137} \mathrm{Cs} 662 \mathrm{keV}$ peak. Resolution at the highest rates is around $8 \mathrm{keV}$. Previous world's-best performance from the ADONIS system [Barat] plateaued at about $90 \mathrm{kcps}$ output rate for $662 \mathrm{keV}$ gamma-rays whereas the UHRGe system operates at output rates as high as $425 \mathrm{kcps}$. Further improvements are expected when detectors with shorter charge collection times are employed, allowing for reduction in the filter gap time from $800 \mathrm{~ns}$ to $\sim 300 \mathrm{~ns}$; currently the gap time is a significant fraction of the total filter length at high rates. 
PNNL-23084

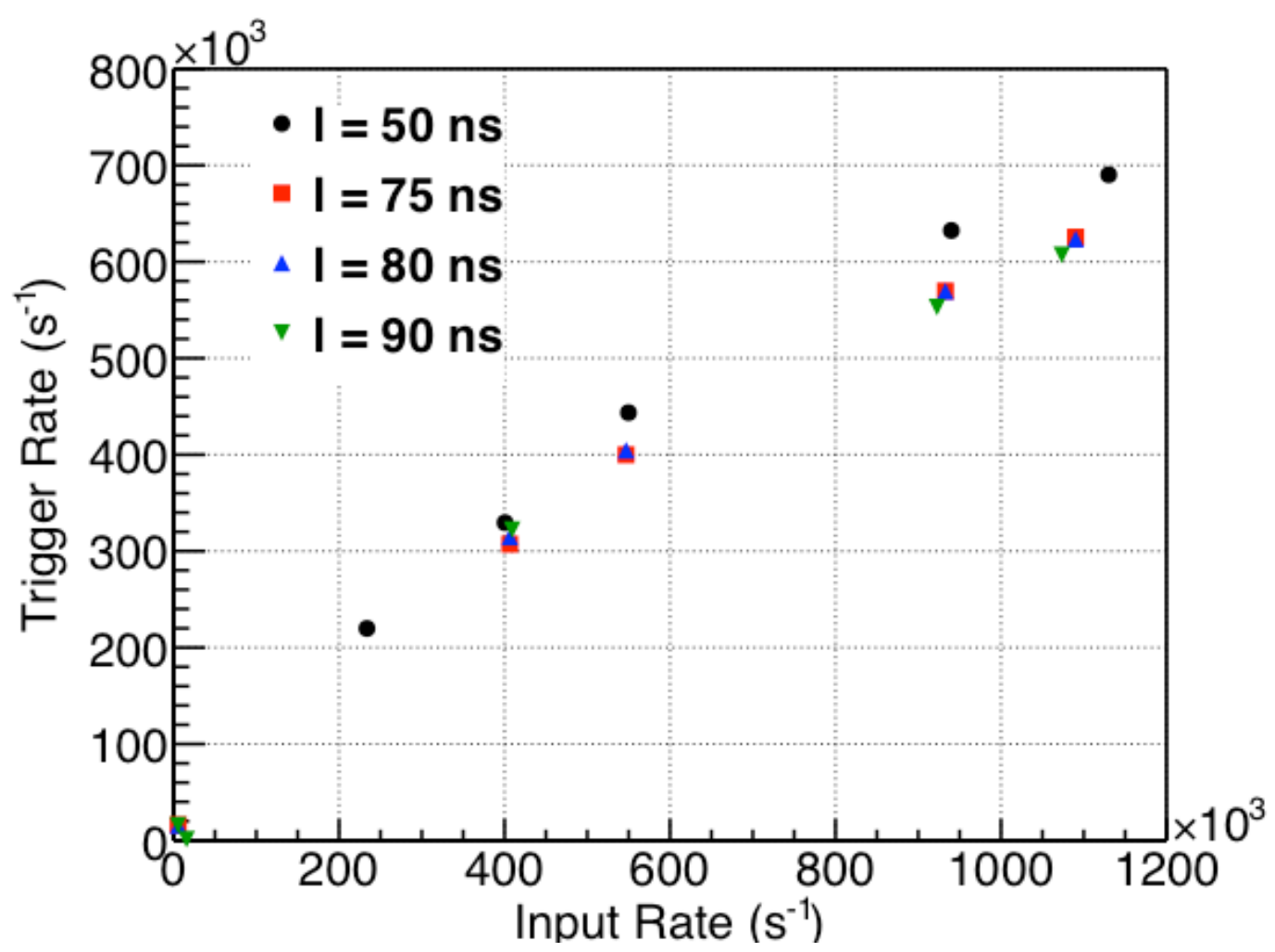

Figure 7. Trigger rate versus input count rate.

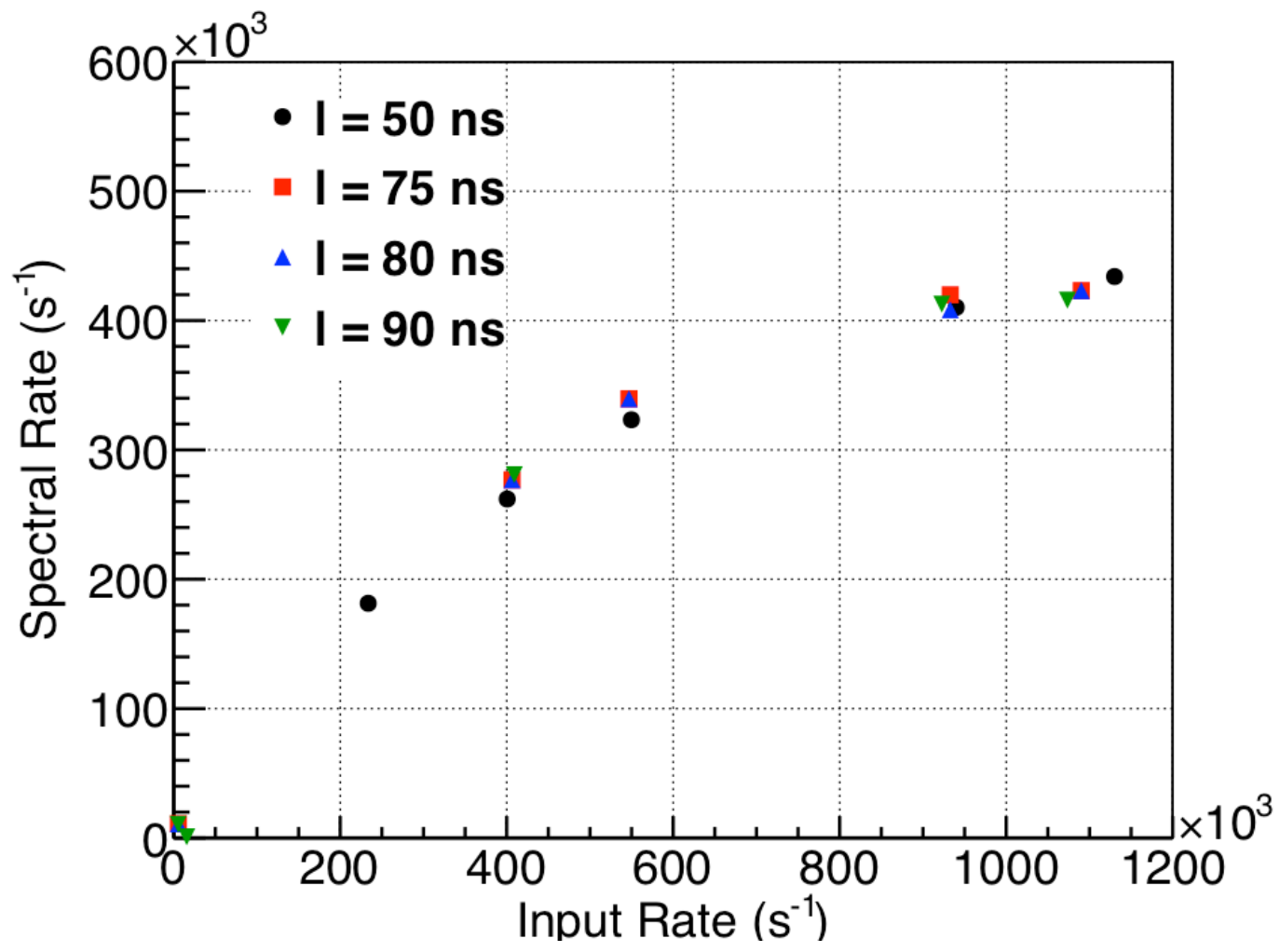

Figure 8. Output rate (to energy histogram) versus input count rate. 
PNNL-23084

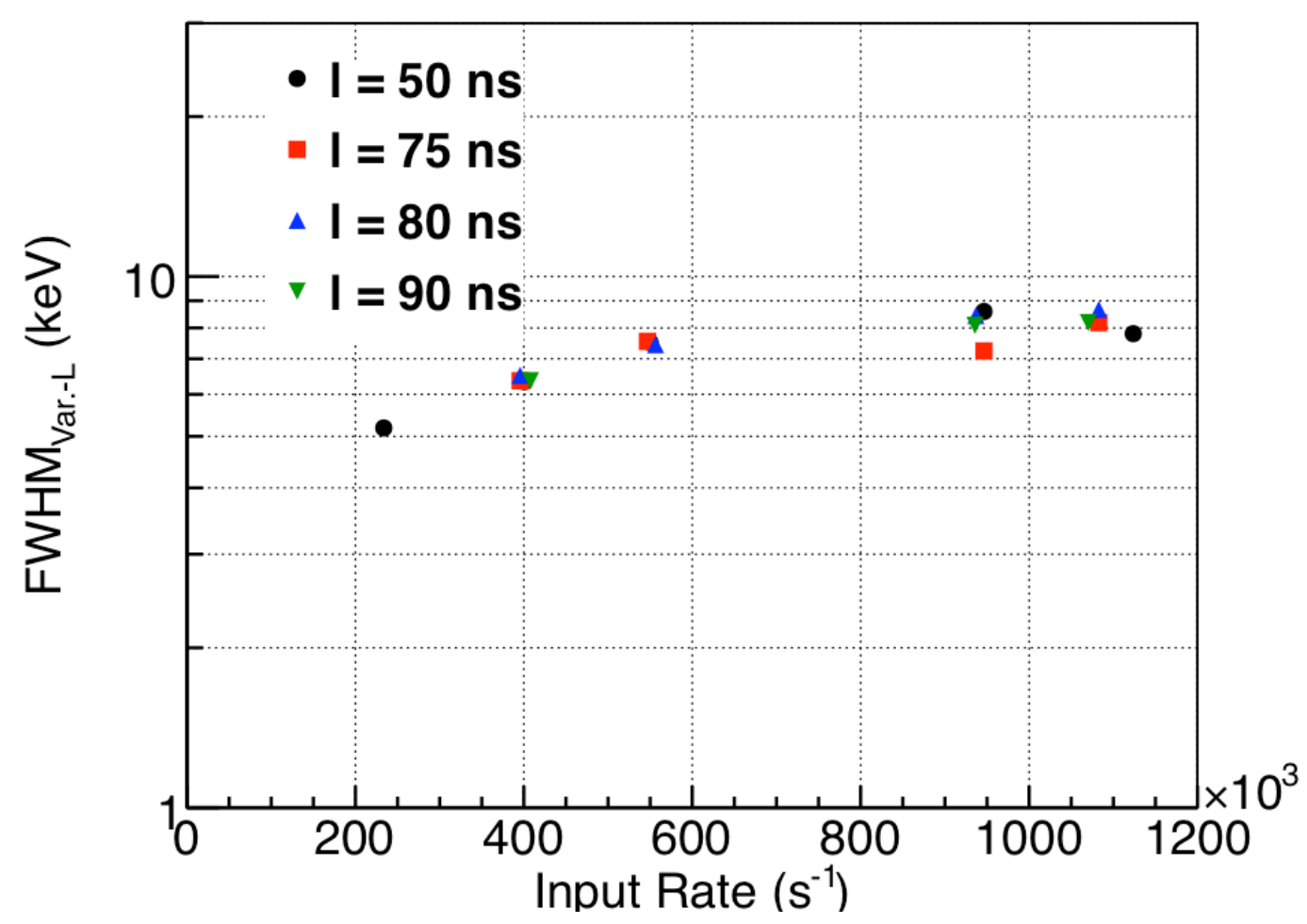

Figure 9. Energy resolution versus input count rate.

\subsection{Results obtained with real time signal processing in the FPGA}

The FPGA implementation of the parallel trapezoidal filters described earlier was tested with various combinations and geometries of 1,10 , and $800 \mathrm{uCi}{ }^{137} \mathrm{Cs}$ sources, providing gamma rates from 2.6 up to $500 \mathrm{kcps}$. List mode data was read from the FPGA via the PCI Express bus and an energy spectrum created (see Figure 10 and Figure 11). Different sets of energy filter lengths were used for gamma rates below and above $100 \mathrm{kcps}$; the specific filter lengths are listed in Table 1 . The $662 \mathrm{keV}{ }^{137} \mathrm{Cs}$ peak in the resulting spectra was fit and the FWHM measured as a determination of energy resolution.

Table 1. Filter sets used in this analysis.

\begin{tabular}{||c|c|c||}
\hline \hline & $\begin{array}{c}\text { Filter set A } \\
\text { (< 100 kcps input) }\end{array}$ & $\begin{array}{c}\text { Filter set B } \\
\text { (>= 100 kcps input) }\end{array}$ \\
\hline \hline 1 & 4.096 us & $512 \mathrm{~ns}$ \\
\hline 2 & 8.192 us & 1.024 us \\
\hline 3 & 12.288 us & 2.048 us \\
\hline 4 & 16.384 us & 4.096 us \\
\hline
\end{tabular}


Energy resolutions at $662 \mathrm{keV}$ for both sets of filters are listed in Table 2; these values were formed by taking the energy measurement from the longest filter that completed for each trigger, and so represent an aggregate of the various filter lengths. A nonlinear degradation in energy resolution was observed between the two highest rates ( $425 \mathrm{kcps}$ and $500 \mathrm{kcps}$ ) in the FPGA analysis. This is not observed in offline analyses so it is likely to be an artifact introduced in real-time event processing on the FPGA.

Additionally, the real-time DC offset calculation used in the offline analysis is not yet implemented in the FPGA. From off-line analysis we know that the DC level shifts as the rate is increased. A DC shift translates directly into the apparent width of a monoenergetic spectral peak, and thus degrades resolution. This is also the cause of the low-side tails on the $662 \mathrm{keV}$ peak that are visible in the spectra. Further adjustment of the DC offset in the FPGA requires a second fast triangle filter, which is used along with the trigger filter to determine the DC level in the presence of extreme pileup where the baseline is never sampled. Energy resolution is expected to more closely resemble the off-line analysis results at high rates once this correction is implemented.

Table 2. Energy resolution vs. rate for the algorithm in FPGA.

\begin{tabular}{||c|c|c||}
\hline \multirow{4}{*}{ Filter Set } & Input Rate (Kcps) & $\begin{array}{c}\text { Energy resolution } \\
\text { (FWHM, keV) }\end{array}$ \\
\hline \hline \multirow{4}{*}{ A } & 5.0 & 2.09 \\
\cline { 2 - 3 } & 32.4 & 2.43 \\
\cline { 2 - 3 } & 54.3 & 3.29 \\
\cline { 2 - 3 } & 73.2 & 4.20 \\
\cline { 2 - 3 } & 100.3 & 4.23 \\
\hline \multirow{3}{*}{ B } & 100.3 & 3.69 \\
\cline { 2 - 3 } & 195 & 5.58 \\
\cline { 2 - 3 } & 425 & 10.15 \\
\cline { 2 - 3 } & 500 & 21.49 \\
\hline
\end{tabular}

Figure 12 and Figure 13 demonstrate how different filter lengths become more appropriate at different rates. The fraction of events for which each filter is used is plotted in the top half of each figure, and the corresponding energy resolution in the bottom half. Note how as rates increase the longer filter fractions become smaller and shorter filters start dominating the accepted collection. Also note that while at low rates the longest filter consistently produces the best energy resolution, it can be seen that as rates increase, shorter filters begin to consistently produce better results, even when the trigger rate appears to favor longer filters.

Further improvements to the FPGA implementation (beyond the additional DC-offset correction method described above to improve performance at higher rates) would include the addition of more energy filters for each channel, and output of the energy measurements from all filters for each event, to take advantage of the observation that the longest filter possible does not always provide the best resolution. 

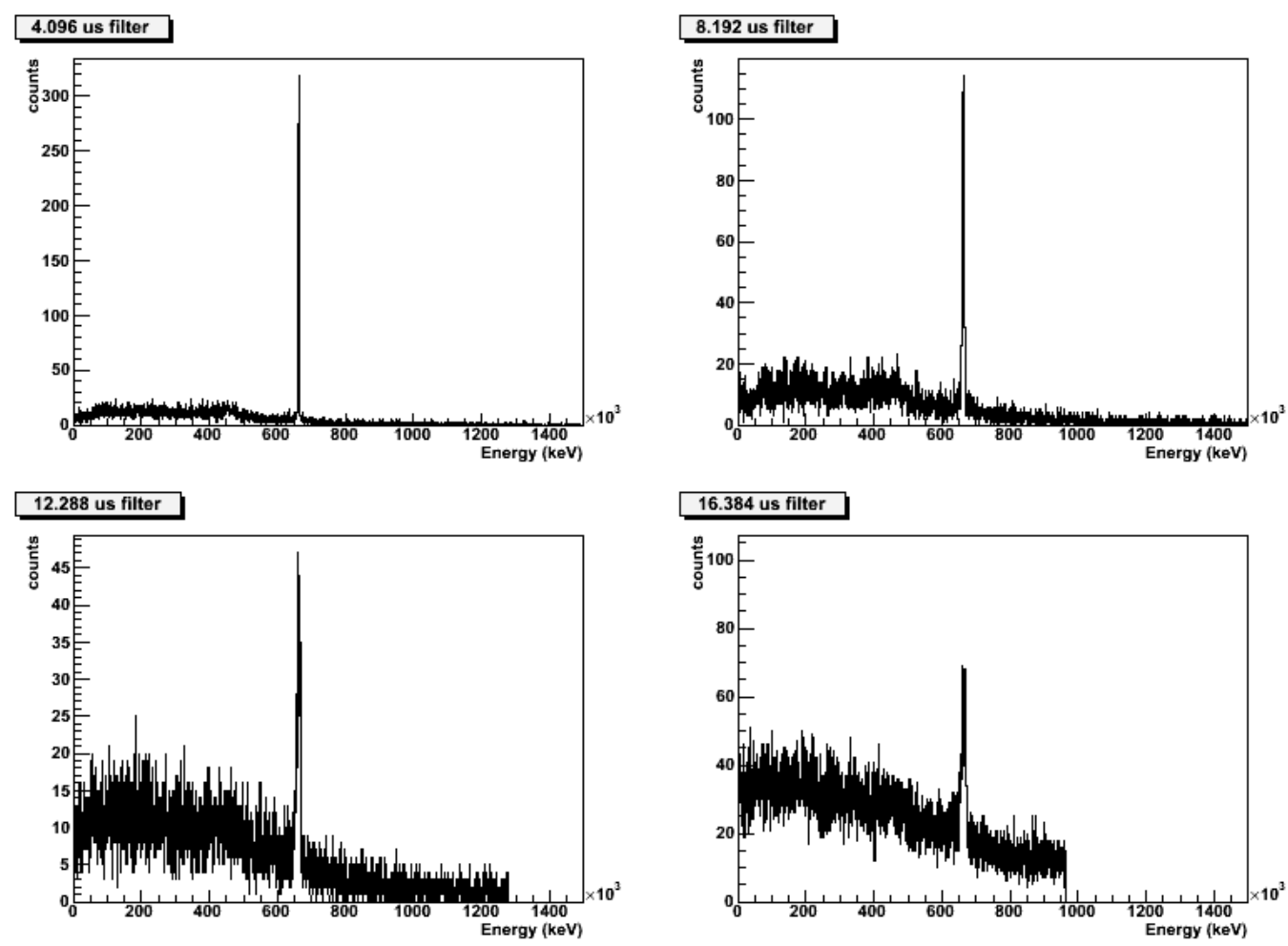

Figure 10. Spectrum at $73.7 \mathrm{kcps}$ input gamma rate, using filter set A.
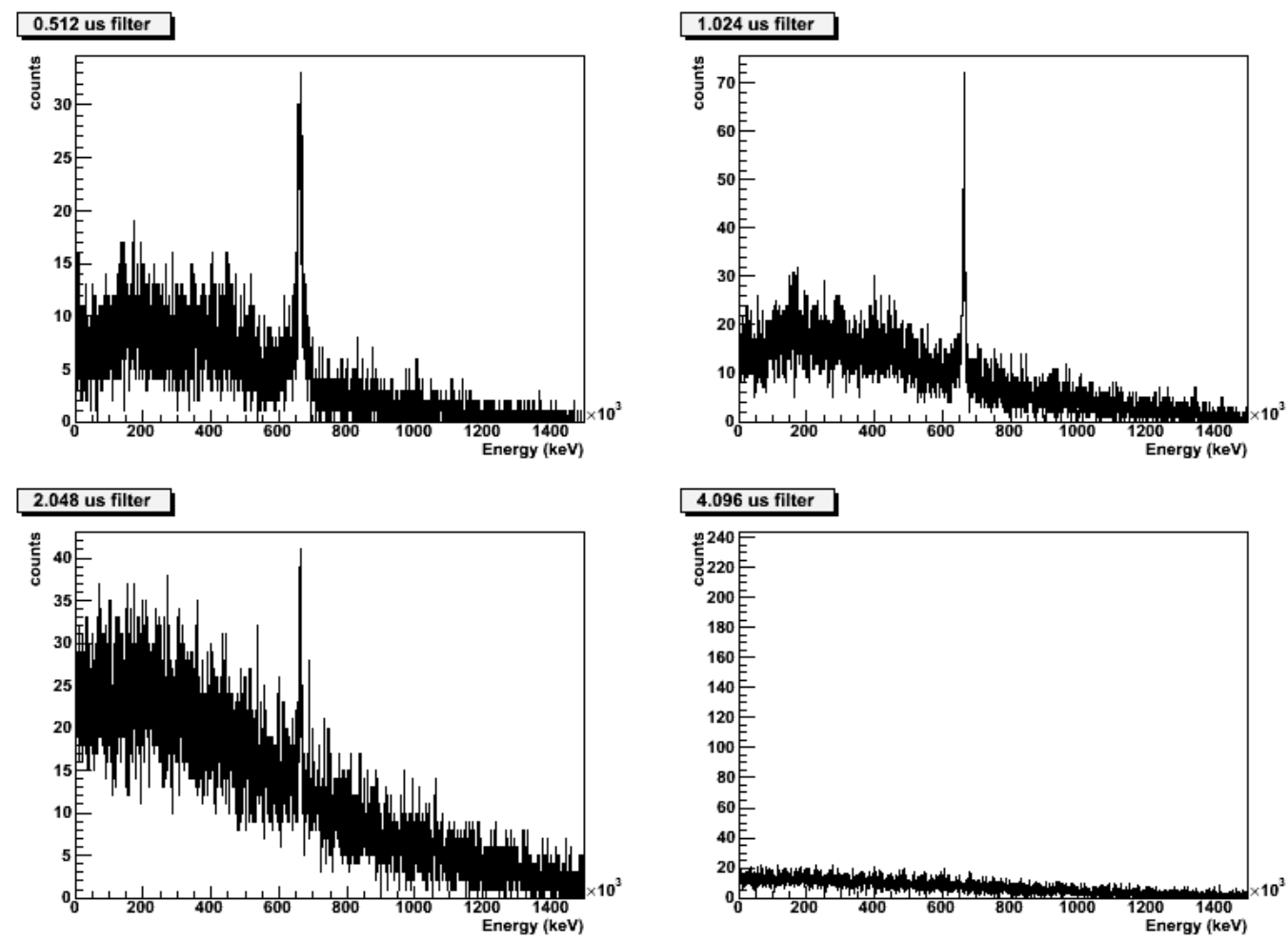

Figure 11. Spectrum at $425 \mathrm{kcps}$ input gamma rate, using filter set B. 
PNNL-23084
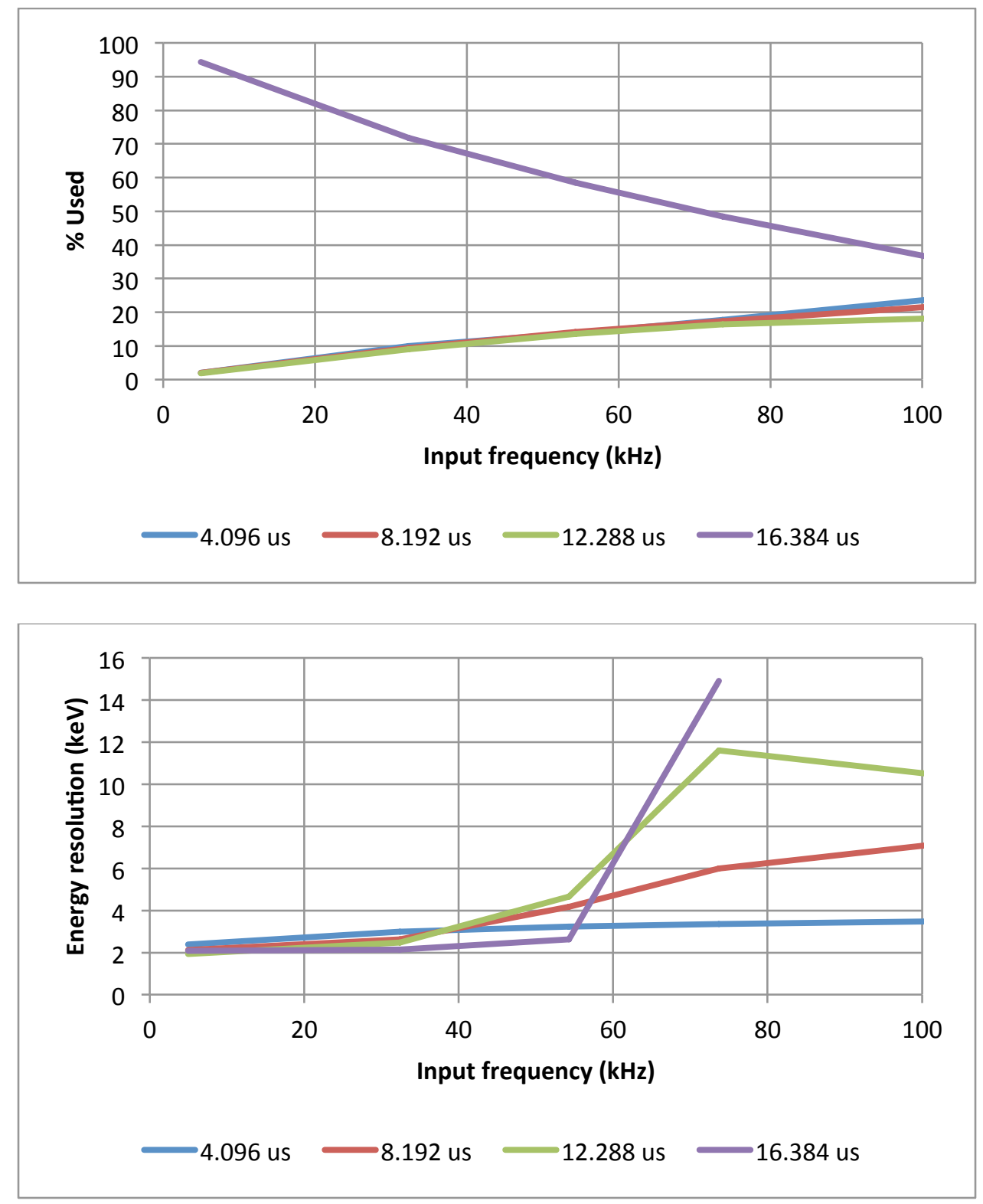

Figure 12. Frequency of use and energy resolution for filter set $A$ at lower rates. The curves represent filters with rise times as shown in the caption with all having the same gap time of $800 \mathrm{~ns}$. 
PNNL-23084
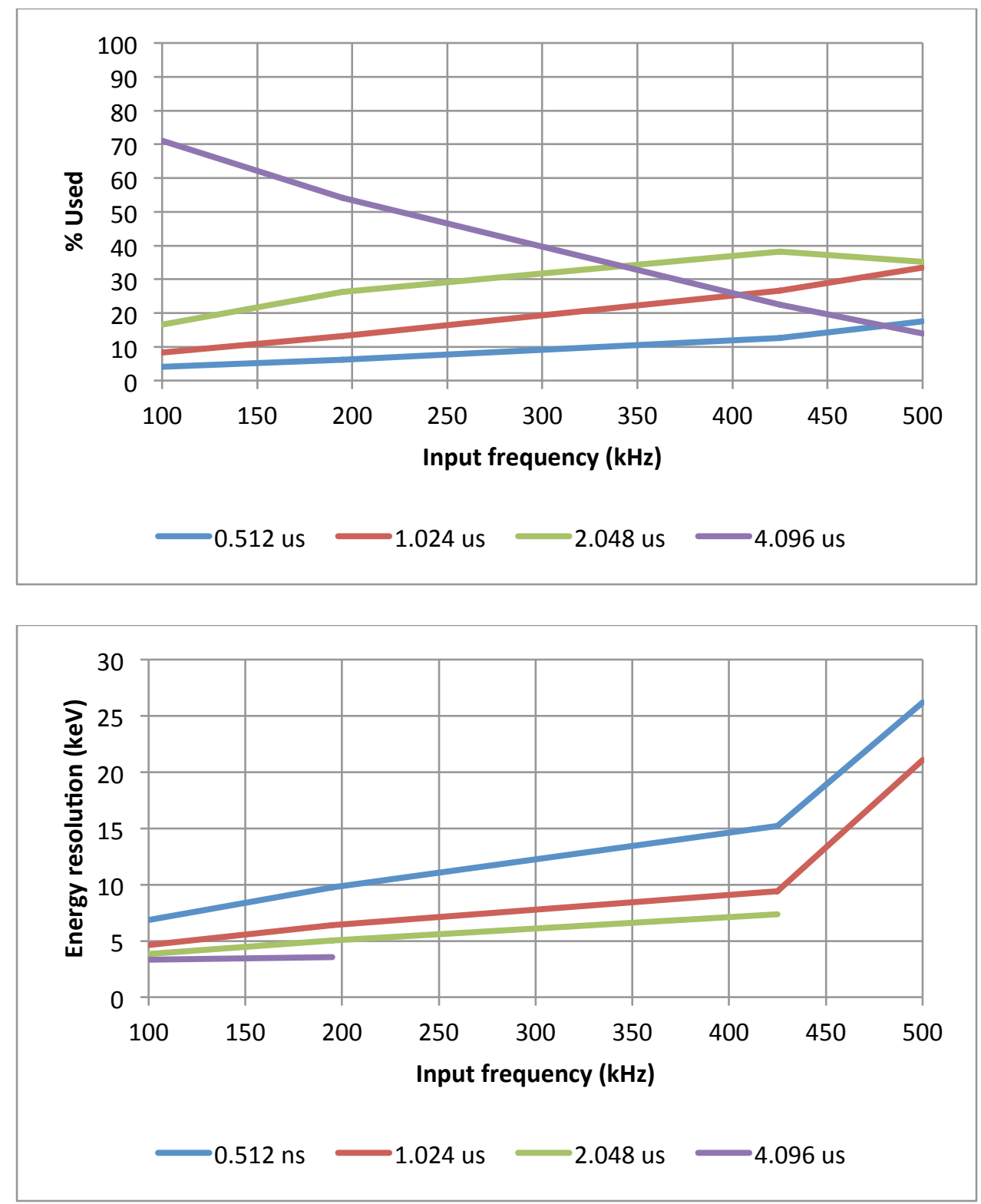

Figure 13. Frequency of use and energy resolution for filter set B at higher rates. The curves represent filters with rise times as shown in the caption with all having the same gap time of $800 \mathrm{~ns}$. 


\subsection{Conclusions}

The UHRGe project has developed a test bed of HPGe detectors with modified and custom designed analog electronics coupled to modern high-speed digitizers running custom waveform analysis in real time to extract list mode data for gamma-ray analysis. The real-time analysis provides time and energy for each energy deposition that will allow future integration of event building in multi-channel systems. The rate capability of the system has been tested with offline analysis at input count rates in excess of 1 Mcps with $>40 \%$ throughput and reasonable energy resolution ( $\sim 8 \mathrm{keV} \mathrm{FWHM}$ at $662 \mathrm{keV})$. The output count rate of $425 \mathrm{kcps}$ is 5 times higher than the prior worlds-best from the ADONIS system [Barat]. The waveform analysis tools developed offline have in large part been ported to the digitizer FPGA and operation at input rates up to $425 \mathrm{kcps}$ return performance similar to the offline analysis, though a critical DC baseline calculation tool is not yet implemented on the FPGA and hence the energy resolution observed is degraded from what can be achieved with the offline code. At low rates where this correction is not required, the online analysis running in the FPGA matches the energy resolution performance of the offline code. Journal manuscripts of the details of the analysis techniques and the real-time signal processing will be submitted for publication soon.

In addition, the project has explored a novel 7-pixel HPGe detector produced by PhDs Co. After considerable study, we have found that the detector has a narrow range of bias voltage settings in which it operates ideally and that this is considerably lower than the voltage initially suggested by the vendor. When operated at this voltage (-200 V bias) the detector has good energy resolution for collimated sources, but non-ideal resolution for flat-field irradiation. Many interesting features have been observed in the detector ranging from the unusual behavior as additional voltage is applied to interesting shifts in fullenergy peaks (without additional tails) for events that share charge between pixels versus events with all charge deposited on one pixel. The results of these studies will be submitted for publication in the archival journal Nuclear Instruments and Methods.

In summary, the project has achieved a $>5$-fold improvement in output count rate compared to the previous worlds-best from the ADONIS detector. With segmentation of the HPGe detector, faster charge collection time HPGe sensors, and further improvements in signal processing there are straightforward paths to $>1$ Mcps output rate and the possibility for much higher rates. This positions the technology well for further development as part of a Safeguards measurement system for spent fuel assay using the delayed gamma technique for long cool down times and for direct passive assay at short cooling times. For the very challenging delayed gamma assay the signal of interest is roughly 1 part in $10^{8}$ of the passive background, placing a very high premium on output count rate so that one can collect a few hundred or thousand of the gammas of interest is a reasonable period of time ( $\sim 1$ day or less). 


\subsection{References}

Nocente, M, M Tardocchi, A Olariu, S Olariu, RC Pereira, IN Chugunov, A Fernandes, DB Gin, G Grosso, VG Kiptily, A Neto, AE Shevelev, M Silva, J sousa, and G Gorini. 2013. "High Resolution Gamma Ray Spectroscopy at $\mathrm{MHz}$ Counting Rates with $\mathrm{LaBr}_{3}$ Scintillators for Fusion Plasma Applications." IEEE Transactions on Nuclear Science 60(2-2):1408-15.

Jordanov, VT, and GF Knoll. 1994. "Digital Synthesis of Pulse Shapes in Real-Time for High-Resolution Radiation Spectroscopy." Nuclear Instruments \& Methods in Physics Research Section aAccelerators Spectrometers Detectors and Associated Equipment 345(2):337-45. 10.1016/01689002(94)91011-1.

Jordanov, VT, GF Knoll, AC Huber, and JA Pantazis. 1994. "Digital-Techniques for Real-Time Pulse Shaping in Radiation Measurements." Nuclear Instruments \& Methods in Physics Research Section a-Accelerators Spectrometers Detectors and Associated Equipment 353(1-3):261-64. 10.1016/0168-9002(94)91652-7.

Wood, LS, JE Fast, DC Rodriguez, MS Taubman, BA Vandevender, C Wilen, and ME Wright. 2013. "A High-Speed Germanium Detector Filter Algorithm Implemented in FPGA." To be submitted to IEEE Transactions on Nuclear Science.

Barat, E et al., “ADONIS: a new system for high count rate HPGe $\gamma$ spectrometry,” IEEE Nuclear Science Symposium Conference Record, Vol. 2 (2006). 



\section{Distribution}

No. of

\section{Copies}

\# Name

Organization

Address

City, State and ZIP Code

\# Organization

Address

City, State and ZIP Code

Name

Name

Name

Name

Name (\#)

\# Name

Organization

Address

City, State and ZIP Code
No. of

\section{Copies}

(1)




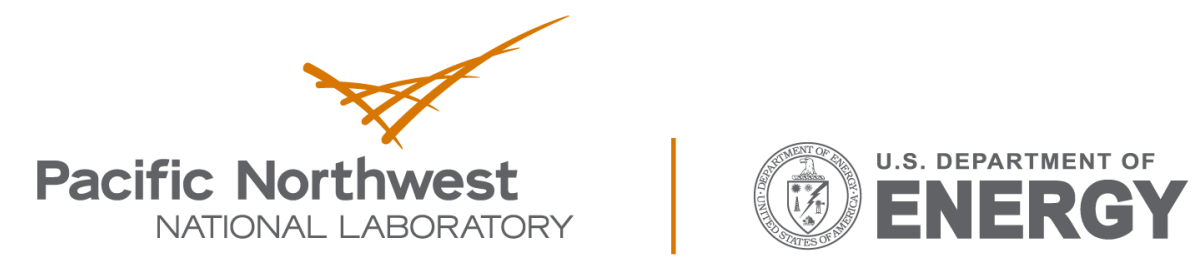

Proudly Operated by Battelle Since 1965

902 Battelle Boulevard

P.O. Box 999

Richland, WA 99352

1-888-375-PNNL (7665)

www.pnnl.gov 